\title{
Sistematização de experiência em educação sanitária no reassentamento urbano Porto Novo
}

\author{
Systematization of experience in health education in the Porto Novo urban \\ resettlement
}

Márcia Monks Jantzen ${ }^{1}$, Marilise Mesquita ${ }^{2}$, Graziella Chaves Trevilato ${ }^{3}$, Luiza de Holleben Saraiva ${ }^{4}$, Michelle da Silva Shons ${ }^{5}$, Michelle Petersen ${ }^{6}$

1 Docente. Universidade Federal do Rio Grande do Sul (UFRGS), Brasil. E-mail: marcia.jantzen@ufrgs.br

2 Docente. Universidade Federal do Rio Grande do Sul (UFRGS), Brasil. E-mail: marilise.mesquita@ufrgs.br

3 Aluna de Graduação. Universidade Federal do Rio Grande do Sul (UFRGS), Brasil. E-mail: grazy.trevilato@gmail.com

4 Aluna de Graduação. Universidade Federal do Rio Grande do Sul (UFRGS), Brasil. E-mail: luiza.holleben@gmail.com

5 Aluna de Graduação. Universidade Federal do Rio Grande do Sul (UFRGS), Brasil. E-mail: schonssaude@gmail.com

6 Aluna de Graduação. Universidade Federal do Rio Grande do Sul (UFRGS), Brasil. E-mail: mibeckerpetersen@gmail.com

Recebido em: 31/03/2015 | Aprovado em: 22/10/2015

DOI: 10.12957/interag.2016.15900

\section{Resumo}

O presente trabalho documenta as experiências vividas por uma equipe composta por um grupo interdisciplinar de docentes e discentes da Universidade Federal do Rio Grande do Sul (UFRGS), entre os anos de 2011 e 2013, em um reassentamento urbano. Os objetivos da presente ação de extensão foram: proporcionar aos alunos um crescimento acadêmico, sensível as demandas locais; divulgar conhecimentos sobre formas de prevenção de DTAs e outras zoonoses; elaborar um material educativo de acordo com as necessidades desta comunidade. Foi realizado um inquérito domiciliar, onde os moradores foram questionados sobre os hábitos de aquisição, manipulação e armazenamento de alimentos, e foram dadas orientações sobre as doenças transmitidas pelos alimentos (DTAs). Ao mesmo tempo, buscou-se promover a saúde através da difusão de novos conhecimentos relativos à aquisição, à higiene e ao preparo dos alimentos. Os dados foram submetidos a uma análise descritiva. Durante todo o período da ação de extensão, o contato dos alunos dos diferentes cursos da área da saúde fol significativo, e houve a divulgação do médico veterinário como participante do controle sanitário na área de alimentos e a importância do consumo de alimentos inspecionados para a saúde da comunidade. Os resultados foram utilizados como base teórica para a elaboração de material educativo e informativo, atendendo às necessidades locais.

Palavras-chave: reassentamento urbano; doenças

\begin{abstract}
This paper documents the experiences of a team composed of an interdisciplinary group of professors and students from the Federal University of Rio Grande do Sul (UFRGS) in an urban resettlement between 2011 and 2013. The objectives of the University Community Project were: to promote academic growth to students while providing for local needs; to disseminate knowledge about ways to prevent foodborne illnesses and other zoonoses; and, to elaborate an educative material summarizing the educational actions in the community. A household survey was carried out, in which the residents were asked about their habits of acquisition, handling and storage of food, in addition to receiving guidance on foodborne diseases. At the same time, we sought to promote health through the dissemination of new knowledge about the acquisition, hygiene and preparation of food. We submitted the data to descriptive analysis. Throughout the period of the study, contact between students from different health courses was significant. Furthermore, we advertised the veterinarian as a participant from the sanitary control of food and the importance of the consumption of inspected food products to public health. The results were used as a theoretical basis for the development of educational and informational material, taking into account local needs.
\end{abstract}

Keywords: urban resettlement; foodborne diseases; 
transmitidas por alimentos; sistematização de systematization of experiences. experiências.

Área temática: Saúde.

Linha de extensão: Segurança alimentar e nutricional.

\section{Introdução}

É possível observar nas periferias das grandes cidades, em comunidades sem infraestrutura e equipamentos urbanos adequados, o comércio de produtos de origem animal sem inspeção, além da criação de animais domésticos no entorno das casas (porcos, galinhas, patos, etc). Segundo Amson et al. ${ }^{1}$, a maior incidência de surtos alimentares acontece nos próprios domicílios e são associados a alimentos de origem animal, tais como carnes, leites, ovos e seus derivados. Isso ocorre por falta de informação dos consumidores sobre higiene na manipulação dos alimentos e o correto armazenamento, principalmente no que diz respeito à temperatura e tempo de estocagem. É frequente a aquisição de produtos sem identificação, sem data de fabricação ou validade vencida e que assim colocam o alimento e a saúde do consumidor em risco ${ }^{2}$.

A Vila Dique de Porto Alegre, comunidade alvo deste estudo, foi removida da sua área original de ocupação irregular, a qual iniciou sua formação na década de 70, com pessoas provenientes das mais diversas regiões do interior do estado do Rio Grande do $\mathrm{Sul}^{3}$. Este local situava-se às margens de um arroio, sem esgotamento sanitário adequado, sem infraestrutura urbana, o que trouxe vários problemas de saúde para a população local, bem como contaminação ambiental, devido em grande parte à concentração de poluentes orgânicos e resíduos sólidos domésticos expostos ao meio ambiente $^{4}$. Esta comunidade começou a ser removida em 2009 para um novo local, construído e estruturado com equipamentos urbanos, para receber as famílias e até o início de 2012, cerca de 1.000 famílias já haviam sido reassentadas.

Tendo em vista a baixa escolaridade e nível socioeconômico desta comunidade, onde um número expressivo de moradores eram catadores de materiais recicláveis ${ }^{5}$, e também pelo fato da comunidade ter adquirido hábitos e costumes típicos de uma 
população sem acesso à infraestrutura urbana adequada, foi desenvolvido este trabalho. A ação buscou identificar a percepção dos moradores do Conjunto Habitacional Porto Novo quanto às doenças transmitidas por alimentos (DTAs). Além disto, as atividades envolviam a discussão de temas para a promoção da saúde daquela comunidade, através da difusão de novos conhecimentos relativos à aquisição, à higiene e ao preparo dos alimentos. Segundo Lermen e Fisher ${ }^{6}$ um maior grau de escolaridade gera um maior senso crítico sobre os fatores ambientais, e em $89 \%$ dos casos, a principal fonte de conhecimento sobre o meio ambiente vem da escola; por isso, a importância de ações de educação ambiental no âmbito do ensino não formal ${ }^{7}$.

Para relatar as ações de extensão, pode-se utilizar uma metodologia ainda pouco conhecida, a da Sistematização de Experiências (SE), desenvolvida na década de 80, no México, como uma prática de educação popular. Naquele momento, profissionais vinculados ao Centro de Estudos do Terceiro Mundo (CEESTEM) começaram a sentir a necessidade de recuperar e comunicar experiências vividas, gerando lições que não estavam sendo devidamente divulgadas e replicadas. Segundo Martinic ${ }^{8}$, a sistematização de experiências pode ser definida como um processo de reflexão que pretende ordenar e organizar o que têm sido a trajetória, os processos, os resultados de um projeto, buscando nessa dinâmica as dimensões que podem explicar o curso que assumiu o trabalho realizado. A SE reúne a maior quantidade possível de opiniões para que se possa considerar de forma efetiva as experiências e os pontos de vista das diferentes pessoas envolvidas com a ação ${ }^{9}$.

Os objetivos da presente ação de extensão foram: proporcionar aos alunos um crescimento acadêmico, sensível às demandas locais; divulgar conhecimentos sobre formas de prevenção de DTAs e outras zoonoses; elaborar um material educativo de acordo com as necessidades desta comunidade.

\section{Material e Métodos}

A equipe de extensionistas da Universidade Federal do Rio Grande do Sul, que realizou a presente ação, foi composta por 6 alunas da graduação dos cursos de Fisioterapia, Medicina Veterinária, Saúde Coletiva e Enfermagem, e duas professoras dos cursos de Saúde Coletiva e Medicina Veterinária. 
Foi utilizada a técnica de sistematização de experiências como metodologia de descrição da ação. As fontes de informações utilizadas para a construção da presente SE, além dos relatos dos componentes da equipe de trabalho, também se constituíram de artigos publicados ou aguardando publicação sobre as ações desenvolvidas, publicações de sistematizações de experiências semelhantes em variadas populações carentes ou rurais, artigos publicados em revistas e periódicos atualizados e guias de sistematização de experiências. A referência usada para a SE foi a descrita por Holliday e $\mathrm{CIDAC}^{10}$, que organiza a proposta metodológica em cinco tempos. O primeiro tempo depende do autor da sistematização ter vivenciado a experiência a ser sistematizada, o que equivale a ter participado ativamente da experiência e ter registros dela. $\mathrm{O}$ segundo tempo é a elaboração das perguntas iniciais, através da definição do objetivo, delimitação do objeto a sistematizar, identificação do eixo da sistematização, fontes de informação a serem utilizadas e o procedimento a ser seguido. O terceiro tempo compreende a recuperação do processo vivido, onde se procura reconstruir a história, ordenando e classificando a informação. Esse passo é a Sistematização propriamente dita, sendo o desenvolvimento do trabalho em questão. O quarto tempo é uma reflexão, buscando entender, analisar e sintetizar o processo, através de uma interpretação crítica deste. Uma discussão sobre os resultados obtidos ao final do projeto cumpre essa função. O tempo final consiste da formulação de conclusões e comunicação das aprendizagens.

A coleta de dados se deu por meio da realização de entrevistas, utilizando-se como instrumento um questionário elaborado pelas alunas e professoras. Após o término da elaboração dos questionários, realizou-se o mapeamento do Conjunto Habitacional, descrevendo todas as casas das quadras A, B, C e D, para o controle de quais casas já haviam sido visitadas. Os mapas foram confeccionados a partir de plantas baixas fornecidas pela assistente social coordenadora da equipe de apoio aos moradores da empresa responsável pela construção do loteamento. As visitas nos domicílios tiveram início em novembro de 2011 e finalizaram em dezembro de 2012, em uma amostra do tipo aleatória, que abrangeu todas as quadras da comunidade (quadras A, B, C e D). A SE e o material educativo foram elaborados e concluídos no período de 2013 a 2015. 
O questionário aplicado continha 45 perguntas do tipo fechadas e em alguns casos com múltiplas respostas, sobre os critérios utilizados no momento da aquisição, preparo e consumo dos alimentos. Além disto, algumas perguntas provocavam a explicação da importância do médico veterinário no cuidado sanitário dos alimentos de origem animal, e de determinadas zoonoses adquiridas pela carne e leite. As perguntas selecionadas para análise dos dados foram: O sr(a) compra carnes (boi, frango, suíno, pescados) vendidas por ambulantes? O $\operatorname{sr}($ a) utiliza ovos com a casca rachada e/ou suja de fezes? $\mathrm{O}(\mathrm{a}) \operatorname{sr}(\mathrm{a})$ costuma preparar maionese em casa? Se prepara, utiliza ovos crus? Caso prepare a maionese com ovo cru, costuma consumi-la imediatamente? Quando prepara maionese com ovo cru, consome o que sobra em uma próxima refeição? Qual(ais) aspecto(s) pode(m) apresentar um alimento que oferece risco à saúde? Para $\mathrm{o}(\mathrm{a}) \operatorname{sr}(\mathrm{a})$, as doenças de origem alimentar (toxinfeccções) podem causar que tipo de sintomas? $\mathrm{O}(\mathrm{a}) \operatorname{sr}(\mathrm{a})$ sabia que as carnes mal cozidas podem transmitir doenças para o humano? $\mathrm{O}(\mathrm{a}) \operatorname{sr}(\mathrm{a})$ sabia que leite sem tratamento industrial (comprado diretamente do leiteiro) pode transmitir doenças para as pessoas? $\mathrm{O}(\mathrm{a}) \operatorname{sr}(\mathrm{a})$ sabe que os alimentos de origem animal passam por inspeção de um veterinário antes de chegar ao comércio?

As entrevistas foram realizadas nos domicílios no período da tarde. As casas onde havia pessoas adultas e dispostas a participar, eram então visitadas e sempre uma dupla, alunos e/ou professores da equipe do projeto, realizava as perguntas. As perguntas eram lidas pelo entrevistador para o entrevistado, de maneira clara e objetiva, a fim de incluir também aqueles respondentes que não sabiam ler. As anotações eram realizadas na planilha de respostas pelo entrevistador, no momento da entrevista e, posteriormente, tabuladas em planilha eletrônica. O critério de inclusão na pesquisa foi o entrevistado ser o responsável pela aquisição e elaboração dos alimentos nos domicílios e o critério de exclusão foi indivíduos menores de 16 anos de idade. Todos os entrevistados assinaram um Termo de Consentimento Livre e Esclarecido, redigido em duas vias, conforme as normas expressas na resolução $\mathrm{n}^{\circ} 466$ de $2012^{11}$, permanecendo uma delas com o sujeito da pesquisa, que lhe garante a ocorrência de riscos mínimos no desenvolvimento do projeto, a liberdade de abandonar a pesquisa a qualquer momento e sem prejuízo para si; a privacidade; o anonimato; o compromisso com a informação atualizada do estudo e a garantia de que todos os seus 
questionamentos serão esclarecidos. O projeto foi submetido e aprovado pelo comitê de ética em pesquisa da instituição envolvida sob o número 20648. Os dados colhidos foram submetidos a uma análise descritiva e após se procedeu a SE.

\section{Resultados e Discussão}

A equipe de trabalho entrevistou 225 famílias do reassentamento. Ao final de cada inquérito, abria-se um espaço para o esclarecimento das questões abordadas, bem como para a solução de eventuais dúvidas dos entrevistados. Depois das primeiras semanas foi notável a mudança de postura das alunas, que começaram a interagir melhor com os moradores. Conforme as visitações tornavam-se mais frequentes, cada entrevista passou a tomar mais tempo, pois os moradores demonstravam mais curiosidade sobre os assuntos abordados, contribuindo com suas experiências pessoais e sanando dúvidas importantes. Enquanto isso, as alunas sentiam-se cada vez mais confiantes, tanto para fornecer informações técnicas, quanto para aprender com os moradores. Nos dias de entrevista ocorria a interação entre o saber popular e o científico, numa troca constante, que trouxe sensibilidade social às alunas do projeto, as quais passaram a perceber as demandas locais, para além do instrumento de pesquisa.

Com relação às respostas obtidas nas entrevistas, quando os moradores foram questionados se compravam carnes e derivados de animais vendidos por ambulantes, apenas $10 \%$ deles responderam que sim. Algumas das justificativas para a não aquisição desses produtos foram pela contaminação “com vermes”, má higiene, exposição às moscas, possibilidade de causar "dor de barriga" e vômitos, não saber a procedência, não saber o prazo de validade, "poder fazer mal às crianças", ou não saber de que animal realmente é a carne. A grande maioria de fato não comprava, porque não confiava na qualidade sanitária dos produtos.

Como o ovo é um alimento que muitas vezes está envolvido em casos de salmonelose, principalmente no preparo de maionese caseira onde se utilizam ovos crus 1, algumas perguntas abordaram o risco de contrair essa infecção alimentar. Com relação à utilização de ovos com casca rachada e/ou suja de fezes, $74 \%$ dos entrevistados responderam não utilizar ovos nestas condições, 23\% responderam que sim e $3 \%$ responderam não saber. Houve diversos relatos de pessoas que afirmaram que quando 
o ovo não está rachado, mas apenas sujo de fezes, costumam apenas lavar antes de utilizar.

Os alimentos contaminados muitas vezes não apresentam alterações no odor nem no sabor, dificultando ao consumidor a identificação de quais alimentos poderiam estar $\operatorname{contaminados}^{12}$. Quando questionados sobre o que seria um alimento que oferece risco à saúde, a grande maioria das pessoas $(84 \%)$ respondeu que seria aquele que apresenta mau cheiro e aspecto desagradável. Porém, 52\% dos entrevistados pensavam que apesar do aspecto agradável, o alimento poderia causar problemas à saúde. Ainda 49\% dos indivíduos afirmaram que pode haver risco quando o alimento não ficou todo o tempo sob refrigeração, revelando que quase a metade dos entrevistados tem consciência da importância desta prática na conservação dos alimentos. Outra pergunta realizada no inquérito estava relacionada à percepção sobre o que são doenças transmitidas por alimentos (DTAs) ou toxinfecções alimentares. Neste quesito, $52 \%$ dos entrevistados responderam que as DTAs podem provocar outros sintomas além de vômitos e/ou diarreia.

Para relacionar os hábitos e costumes com os conhecimentos acerca de segurança alimentar, perguntamos se a pessoa sabia que a carne mal cozida pode transmitir doenças do animal para o humano, e $80 \%$ responderam afirmativamente. Isso é um ponto favorável para se evitar DTAs e zoonoses, embora muitos tenham relatado que tem dessa informação, mas gostam de consumir carnes "mal passadas".

O percentual de indivíduos que responderam saber que a carne sem inspeção pode transmitir doenças do animal para o humano (80\%) foi maior do que quando a pergunta era relacionada ao leite sem inspeção, quando 59\% dos entrevistados afirmaram saber que o leite cru pode trazer doenças. Isso serve como um alerta de que nessa comunidade a crença de que a carne pode ser mais contaminante que o leite existe, indicando assim a necessidade de melhor esclarecimento para a população.

Outra pergunta de cunho informativo diz respeito à atuação do médico veterinário, quando foi perguntado se as pessoas sabiam que os alimentos de origem animal passam por inspeção de médico veterinário antes de chegar ao comércio. Nessa questão, $56 \%$ 
dos entrevistados afirmou desconhecer essa informação, e a ação serviu para difundir a atuação do médico veterinário na vigilância sanitária dos alimentos.

A última etapa do trabalho foi a elaboração de um material impresso contendo noções de educação sanitária sobre alimentos e informações sobre DTAs. Este material foi elaborado a partir dos dados coletados nas entrevistas domiciliares. Após uma análise descritiva das respostas das entrevistas, foi possível perceber quais os principais riscos à saúde a que esta comunidade estava submetida. $\mathrm{O}$ material educativo foi então elaborado para dar respostas e orientações para os problemas específicos observados naquela comunidade. As ilustrações do material impresso foram feitas com desenhos elaborados por uma das alunas do projeto, e com fotos de moradores, devidamente autorizadas, mediante termo de autorização de uso de imagem para fins didáticopedagógicos e acadêmicos. Para este material de educação informal, foi utilizada uma linguagem acessível, tendo em vista o baixo nível de escolaridade observado na comunidade $^{13}$. Foram impressos mil exemplares para serem distribuídos para as famílias do Conjunto Habitacional Porto Novo.

\section{Considerações Finais}

De acordo com a experiência vivenciada pelos alunos e professores nesta ação de extensão, foi possível observar que muitos moradores tinham a percepção de que algumas práticas e costumes poderiam colocar a saúde em risco. Isso foi verificado quando responderam que não costumam comprar carne de ambulantes e que não utilizam ovos com casca rachada. Naquela população, de forma geral, é comum que as pessoas relacionem somente alimentos com aspecto desagradável às doenças. As informações dadas às pessoas da comunidade, de que outras doenças graves ou crônicas podem ser adquiridas através dos alimentos, aconteceram no momento das entrevistas, sob forma de conversa, e serviram para alertar sobre os cuidados a serem tomados com os alimentos dentro de casa.

Durante todo o período da ação de extensão, o contato dos alunos dos diferentes cursos da área da saúde foi significativo. As visitas semanais à comunidade culminaram em reafirmar a Extensão Universitária como processo indispensável à formação dos graduandos, na qualificação do professor e no intercâmbio com a sociedade, o que 
implica em relações multi, inter, transdisciplinar e interprofissional. A ação foi além de pesquisar a percepção dos moradores sobre a saúde alimentar e as informações acerca desse assunto. Houve também a divulgação do médico veterinário como participante do controle sanitário na área de alimentos e a importância do consumo de alimentos inspecionados para a saúde pública da população. Uma ação futura poderia trazer propostas educativas e lúdicas que seriam realizadas com o público infantil, promovendo a internalização de princípios de cuidado com a saúde, para a formação de adultos conscientes.

\section{Agradecimentos}

Agradecemos à Pró-Reitoria de Extensão da UFRGS (PROREXT) pelas bolsas de extensão concedidas, fundamentais para a realização deste projeto.

\section{Referências}

1. AMSON, Gisele Van; HARACEMIV, Sônia Maria Chaves; MASSON, Maria Lucia. Levantamento de dados epidemiológicos relativos às ocorrências/ surtos de doenças transmitidas por alimentos (DTAs) no estado do Paraná - Brasil, no período de 1978 a 2000. Ciênc. Agrotec, v. 30, n. 6, p. 1139-1 145, 2006.

2. PRAXEDES, Paula Cristina Gonzales. Aspectos da qualidade higiênicosanitária de alimentos consumidos e comercializados na comunidade São Remo, São Paulo, Capital. 120f. Dissertação (Mestrado em epidemiologia experimental e aplicada às zoonoses) - Faculdade de Medicina Veterinária e Zootecnia, Universidade de São Paulo. São Paulo, Brasil. 2003.

3. GIL, Carmem Zeli de Vargas. Da Vila Dique ao Porto Novo. Extensão popular, rodas de memórias e remoções urbanas. São Leopoldo: Oikos, 2013.

4. TROLEIS, Adriano Lima. Metrópole de risco: o caso da Vila Dique e do aterro sanitário da zona norte na poluição das águas superficiais e subterrâneas das bacias hidrográficas dos Arroios da Areia e Passo das Pedras/Porto Alegre-RS. Tese de Doutorado do Programa de Pós-graduação em Geografia, Universidade Federal do Rio Grande do Sul; 2009. 
5. ZACARIAS, Inez Rocha; BAVARESCO Caren Serra. Conhecendo a realidade dos catadores de materiais recicláveis da Vila Dique: visões sobre os processos de saúde e doença. Revista Textos \& Contextos, Porto Alegre; 2009, 8 (2): 293-305.

6. LERMEN Helena Salgueiro; FISHER, Paul Douglas. Percepção ambiental como fator de saúde pública em área de vulnerabilidade social no Brasil. Revista de APS. Núcleo de Assessoria e Treinamento de estudos em Saúde, 2010,13 (1): 62-71.

7. DIAS, Genebaldo Freire. Educação Ambiental, Princípios e Práticas. 9 ed. São Paulo: Gaia. 2010.

8. MARTINIC, Sergio. Algunas categorias de análisis para la sistematización. Santiago, Chile. 1984.

9. CHAVEZ-TAFUR, Jorge. Aprender com a prática: uma metodologia para sistematização de experiências. AS-PTA. Brasil. 2007.

10. HOLLIDAY, Oscar Jara; CIDAC (Org). Sistematização de Experiências: aprender a dialogar com os processos. CIDAC. Lisboa. 2007.

11. BRASIL. Ministério da Saúde. Conselho Nacional de Saúde. Resolução no 466, de 12 de dezembro de 2012: diretrizes e normas regulamentadoras de pesquisa envolvendo seres humanos. Brasília: Ministério da Saúde, 2012.

12. FORSYTHE, Stephen J. Microbiologia da segurança alimentar. Porto Alegre: Artmed, 2000. 424 p.

13. JANTZEN, Márcia Monks; MESQUITA, Marilise Oliveira; TREVILATO, Graziella Chaves; SCHONS, Michelle da Silva; SARAIVA, Luiza de Holleben; PETERSEN, Michelle Becker; ESPÍNDOLA, Larissa de Borba; VOGEL, Lucas Werle; SANTOS, Francielle da Silva; PRATES, Cristiane Camargo. Educação para segurança alimentar no Conjunto Habitacional Porto Novo. Porto Alegre: UFRGS, 2015. 40 p. 